\title{
Gallotannin attenuates 2-deoxy-D-glucose-induced dedifferentiation and endoplasmic reticulum stress through inhibition of inositol-requiring enzyme 1 downstream p38 kinase pathway in chondrocytes
}

\author{
SU MIN KIM, YOHAN HAN, SEON-MI YU and SONG JA KIM \\ Department of Biological Sciences, College of Natural Sciences, Kongju National University, \\ Gongju, Chungnam Do 314-701, Republic of Korea
}

Received March 15, 2019; Accepted September 18, 2019

DOI: $10.3892 / \mathrm{mmr} .2019 .10773$

\begin{abstract}
Gallotannin (GT) is a class of polyphenols with antioxidant, anticancer, and antiviral activities. 2-Deoxy-D-glucose (2DG), a glucose-derived molecule, can inhibit glucose metabolism and induce endoplasmic reticulum (ER) stress. GT in primary-cultured chondrocytes enhances expression of type II collagen, an indicator of differentiation, and cyclooxygenase-2 (COX-2), which mediates inflammatory reactions. In contrast, 2DG reduces type II collagen and COX-2 expression while driving ER-stress-induced unglycosylation. In the present study, it was investigated whether GT could attenuate 2DG-induced dedifferentiation and ER-stress. Following treatment with GT and 2DG, chondrocytes were assessed using western blotting, RT-PCR, immunofluorescence, and alcian blue staining. GT restored type II collagen expression that was reduced by $2 \mathrm{DG}$, inhibited ER-stress-induced COX-2 unglycosylation, and induced COX-2 expression. The expression of a glucose-regulated protein, GRP78, which is an indicator of reduced ER-stress, was decreased. To link the GT signaling pathway with pathways that inhibit 2DG-induced dedifferentiation and ER-stress, inhibitors were treated in chondrocytes. The results revealed that, among the different signaling pathways triggered by ER-stress, the p38 kinase pathway was involved in the inositol-requiring enzyme 1 (IRE1) downstream signaling pathway. Following inhibition of the IRE1 pathway, type II collagen expression was increased and COX-2 expression was decreased. In addition, after examining the splicing of $\mathrm{X}$-box binding protein 1
\end{abstract}

Correspondence to: Professor Song Ja Kim, Department of Biological Sciences, College of Natural Sciences, Kongju National University, 50 Gongjudaehak, Gongju, Chungnam Do 314-701, Republic of Korea

E-mail: ksj85@kongju.ac.kr

Key words: gallotannin, 2-deoxy-D-glucose, differentiation, chondrocytes, endoplasmic reticulum stress, p38 kinase, inositolrequiring enzyme 1
(XBP-1) which is dependent on IRE1 activation induced by ER-stress, it was revealed that GT inhibited the increase of XBP-1s after splicing due to 2DG-induced ER stress. GT in chondrocytes inhibited 2DG-induced dedifferentiation and ER-stress-induced COX-2 unglycosylation while regulating differentiation and inflammation via the ER-stress-induced p38 kinase pathway downstream from the IRE1 pathway.

\section{Introduction}

Osteoarthritis (OA) is known to be associated with congenital factors including aging, genetic factors, and acquired factors including trauma and obesity. These factors influence diverse biochemical signaling pathways in chondrocytes leading to unbalanced intracellular metabolic activities (1). OA is caused by cartilage degeneration, which is caused by metabolic imbalance (2). Articular cartilage consists of a high-density extracellular matrix (ECM) comprised of fibronectin, sulfated proteoglycan, and collagen (3). The differentiated chondrocyte phenotype is detected based on type II collagen expression. OA accompanies dedifferentiation and reduction in the level of type II collagen expression (4).

Inflammatory reactions play a crucial role in articular cartilages, and hence, inflammation is a key player in the pathogenesis of OA. Cyclooxygenases (COXs) appear in two protein isoforms, COX-1 and COX-2, among which the latter is produced by inducible enzymes and participates mainly in inflammatory regulation $(5,6)$. COX-2 manifestation is controlled during transcription, post-transcription, and translation (7).

Mitogen-activated protein kinase (MAPK) is the foundation of intracellular signaling that regulates cellular functions including cell survival and apoptosis, mitosis, gene expression, cellular proliferation, inflammatory reaction, and differentiation. These processes are induced by stimulation by external signaling molecules such as cytokines, growth factors, and hormones $(8,9)$. This pathway is found in all eukaryotic cells and presumed to play a pivotal role in cell viability (9). Such signal transduction begins with phosphorylation and activation of MAPK kinase (MAPKK) by a group of protein kinases known as MAPK kinase kinase (MAPKKK), followed by the 
phosphorylation and activation of MAPKs by MAPKKs (10). These MAPKKK and MAPKK enzymes phosphorylate three major proteins of the MAPK family, namely, p38 kinase, extracellular signal-regulated kinase (ERK-1/-2), and c-Jun $\mathrm{N}$-terminal kinase (JNK) (11).

The endoplasmic reticulum (ER) is a huge and dynamic organelle that plays a variety of intracellular roles in calcium preservation, lipid metabolism, and cell signaling. The ER is mainly responsible for protein synthesis and folding. In addition, it facilitates various post-translational modifications that are essential for protein function $(12,13)$. The disruption of ER homeostasis and accumulation of misfolded or unfolded proteins inside the cell causes unfolded protein response (UPR), which is an intracellular signaling system that induces cell damage and apoptosis (14). The mechanism of UPR signaling involves binding of a misfolded protein and glucose-regulated protein $78 \mathrm{kDa}$ (GRP78) to sensor proteins located on the ER membrane including, inositol-requiring enzyme 1 (IRE1), pancreatic ER kinase (PERK), and activating transcription factor 6 (ATF6) (15-17). Among these, IRE1 is the most well-preserved UPR signaling protein in eukaryotes that undergoes autophosphorylation for activation when the level of unfolded proteins increases in the ER to trigger the downstream signaling pathway for $\mathrm{X}$-box binding protein 1 (XBP-1) mRNA splicing. The XBP-1 mRNA after splicing is designated as XBP-1s, while that without splicing is designated XBP-1u. XBP-1s mRNA, which is spliced by IRE1 from XBP-1u mRNA, is expressed as an active XBP-1 protein, which promotes ER-stress-related signaling (18). Persistent ER-stress leads to diseases such as cancer, diabetes, and obesity (19).

2-Deoxy-D-glucose (2DG) is a glucose derivative with $2^{\prime} \mathrm{OH}$ replaced by an $\mathrm{H}$, and this derivative inhibits several metabolic processes, such as glycosylation, leading to ER stress. Treating chondrocytes with 2DG reduces the expression of type II collagen and COX-2, while inducing ER-stress-related unglycosylation (20). Gallotannin (GT) is a hydrolyzable tannin, i.e., a plant polyphenol, which is used as a protective agent in a similar manner to a drug, and exhibits strong activities against cancers, oxidation, viruses, bacteria, and parasites. Treatment of chondrocytes with GT was revealed to promote the expression of type II collagen and COX-2 (21). Although previous studies have examined the independent effects of each of these agents on chondrocytes, the effects of simultaneous treatment with GT and 2DG, in addition to the signaling pathways regulating their activities, have not been adequately investigated (20-22). Thus, the present study aimed to elucidate the effects of treatment with GT and 2DG on chondrocytes and the mechanisms involved.

\section{Materials and methods}

Culture of chondrocytes. The present study was approved by the Ethics Committee of Kongju National University. Fifty specific pathogen-free (SPF) rabbits were sacrificed, one or two rabbits weekly for one year, in compliance with the ethical guidelines, to obtain articular chondrocytes from healthy, normal rabbits (2-weeks old; New Zealand white rabbits; Koatech). The SPF rabbits were supplied from Koatech. Koatech is accredited by the Association for Assessment and Accreditation of Laboratory
Animal Care International (AAALAC), and also complies with the 'Guide for the Care and Use of Laboratory Animals; 8th ed.' (1996, National Research Council). After arrival the condition of the rabbits was monitored every $6 \mathrm{~h}$ and they were sacrificed for the experiment $24 \mathrm{~h}$ upon arrival. The rabbits were anesthetized using 7\% ether and ethically euthanized via cervical dislocation. Ethyl ether was the inhalation anesthetic used, and the concentration was decided based on the contents of a book published in Korea in 1996. Euthanasia of rabbits was confirmed by cardiac arrest (23). The rabbits were fed separately, and had free access to unlimited food and water in an undisturbed and clean environment. The rabbits were housed in a cage that had a 12-h light/dark cycle, and was maintained at a temperature of $\sim 22-25^{\circ} \mathrm{C}$ and a relative humidity ranging from 40 to $60 \%$. Chondrocytes were separated from rabbit articular cartilages, as previously described (24). A piece of cartilage was melted with $0.1 \%$ collagenase for $9 \mathrm{~h}$ in a $36.5^{\circ} \mathrm{C} \mathrm{CO}_{2}$ incubator. Cells ( $5 \times 10^{4}$ cells/dish) were cultured in Dulbecco's modified Eagle's medium (DMEM; Invitrogen; Thermo Fisher Scientific, Inc.) including 10\% (v/v) fetal bovine-calf serum (Tissue Culture Biologicals), penicillin (50 unit/ml), and streptomycin $(50 \mu \mathrm{g} / \mathrm{ml})$.

Treatment of cells. GT (molecular formula: $\mathrm{C}_{76} \mathrm{H}_{52} \mathrm{O}_{46}$; CAS no. 1401-55-4; Sigma-Aldrich; Merck KGaA) and 2DG (molecular formula: $\mathrm{C}_{6} \mathrm{H}_{12} \mathrm{O}_{5}$; CAS no. 154-17-6; Sigma-Aldrich Merck KGaA) were first dissolved with autoclaved triple distilled water and filtered. Chondrocytes were treated with GT $100 \mu \mathrm{M}$ and 2DG $10 \mathrm{mM}$ for $24 \mathrm{~h}$. The concentration and duration of GT and 2DG used in the present study, were determined to best represent the effect of each reagent on chondrocytes based on previous studies (20-22). In addition, SB (CAS no. 152121-47-6; BIOMOL; Enzo Life Sciences), PD (CAS no. 167869-21-8; Calbiochem; EMD/Merck KGaA) and Salubrinal (CAS no. ALX-270-428-M005; Enzo Life Sciences, Inc.) blockers were added $2 \mathrm{~h}$ prior to treatment with GT and 2DG. SB, PD, and Salubrinal inhibit p38 kinase, ERK-1/-2, phosphorylation and dephosphorylation of p-eIF2 $\alpha$.

Western blot analysis. Protein quantification and SDS-PAGE for protein confirmation were conducted from chondrocytes, as previously described (24). The antibodies used $(1: 1,000)$ were as follows: Type II collagen (cat. no. sc-52658); COX-2 (cat. no. sc-1745); GRP78 (cat. no. sc-1050; all from Santa Cruz Biotechnology, Inc.); IRE1 $\alpha$ (cat. no. NB100-2324; Novus Biologicals); p-eIF2 $\alpha$ (cat. no. sc-101670; Santa Cruz Biotechnology, Inc.); ATF6 (cat. no. 70B1413.1; Enzo Life Sciences, Inc.); GAPDH (cat. no. sc-166545; Santa Cruz Biotechnology, Inc.); p-p38 (cat. no. 4511; Cell Signaling Technology, Inc.); p-ERK (cat. no. sc-7383; Santa Cruz Biotechnology, Inc.); anti-rabbit IgG (cat. no. AP132; Sigma-Aldrich; Merck KGaA); anti-goat IgG (cat. no. sc-2354; Santa Cruz Biotechnology, Inc.); and anti-mouse IgG (cat. no. BML-SA204-0100; Enzo Life Sciences, Inc.). An ECL (Daeil Lab Service Co., Ltd.) reagent was used to saw protein bands using the LAS4000 (GE Healthcare Life Sciences).

$R T-P C R$. To confirm the protein expression regulated at the transcriptional level, RNA was extracted from chondrocytes utilizing TRIzol (Invitrogen; Thermo Fisher Scientific, Inc.). 
Furthermore, mRNA was synthesized and amplified by cDNA, and the amount of gene expression was confirmed by electrophoresis on $2 \%$ agarose gel. Each sample was incubated at $95^{\circ} \mathrm{C}$ for $30 \mathrm{sec}, 50^{\circ} \mathrm{C}$ for $30 \mathrm{sec}$, and $72^{\circ} \mathrm{C}$ for $30 \mathrm{sec}$ for 28 cycles. The primer sequences were as follows: Type II collagen sense, 5'-GACCCCATGCAGTACATGCG-3' and antisense, 5'-AGCCGCCATTGATGGTCTCC-3'; COX-2 sense, 5'-CCCTATGAATCGTTCGAGGA-3' and antisense, 5'-GGACAGCCCTTCACATTGTT-3'; XBP-1 sense, 5'-TGG ATGCCATGGTTACTGAA-3' and antisense, 5'-CTGGCA GTTTCTGGAGAAGC-3'; GAPDH sense, 5'-TCACCATCT TCCAGGAGCGA-3' and antisense, 5'-CACAATGCCGAA GTGGTCGT-3'.

Alcian blue staining. Proteoglycans were measured using $0.1 \%$ alcian blue solution (Sigma-Aldrich; Merck KGaA) to determine differentiation of chondrocytes. Cells were reacted with $3.5 \%$ paraformaldehyde for $25 \mathrm{~min}$ and stained as previously described (24). The absorbance was assessed using a microplate reader at $595 \mathrm{~nm}$.

Immunofluorescence staining. Revelation of type II collagen, COX-2 and GRP78 within cells was identified. Cells were fixed and antibodies were attached as previously described (24). All primary antibodies used were the same as those used in the western blotting experiment. The secondary antibodies used were: TRITC conjugated anti-mouse IgG (cat. no. T5393; Sigma-Aldrich; Merck KGaA) for type II collagen, FITC conjugated anti-goat IgG (cat. no. ab6881; Abcam) for COX-2 and TRITC conjugated anti-goat IgG (cat. no. ab150130; Abcam) for GRP78. These secondary antibodies were incubated at room temperature for $2 \mathrm{~h}$. Then, the cells were counterstained with 4'6'-diamidino-2-phenylindole dihydrochloride (DAPI; Invitrogen; Thermo Fisher Scientific, Inc.). Fluorescent images were captured using a BX51 fluorescence microscope (Olympus Corporation).

Transfection. IRE1 siRNA was transfected into chondrocytes using a TurboFect transfection reagent (Thermo Fisher Scientific, Inc.). The primer sequences were as follows: IRE1 siRNA sense, 5'-GAUGUCCCACUUUGUGUCCTT-3' and antisense, 5'-GGACACAAAGUGGGACAUCTT-3'; negative control (NC) siRNA sense, 5'-UUCUCCGAACGUGUCACG UTT-3' and antisense, 5'-ACGUGACACGUUCGGAGAATT-3'.

Statistical analysis. All experimental results were repeated at least 3 times and presented as the mean \pm standard deviation (SD). Data were assayed using one-way analysis of variance (ANOVA) followed by Tukey's post hoc test. $\mathrm{P}<0.05$ was considered to indicate a statistically significant difference.

\section{Results}

GT regulates $2 D G$-induced dedifferentiation, inflammatory reactions, and ER stress. The effects of GT and 2DG treatment for $24 \mathrm{~h}$ on differentiation and inflammatory reactions in cultured chondrocytes were examined. Following treatment, western blot and RT-PCR analyses were performed to detect the changes in the expression of type II collagen (the differentiated phenotype of chondrocytes) and COX-2 (a major protein involved in inflammatory reactions). The results revealed that, after GT and 2DG treatment, the 2DG-induced dedifferentiation was attenuated by GT at the transcriptional level (Fig. 1A and B). GT also attenuated the 2DG-triggered decrease in COX-2 expression and ER-stress-induced unglycosylation, while promoting COX-2 expression. The regulation of ER-stress-induced COX-2 unglycosylation is considered to have occurred at the protein translational or post-translational regulation level and not at the transcriptional level (Fig. 1C and D). The statistical significance of the data from western blotting and RT-PCR were quantified using ImageJ (Fig. 1A-D). These findings provided evidence that $\mathrm{GT}$ regulates $2 \mathrm{DG}$-driven dedifferentiation, inflammatory reactions, and ER stress.

GT regulates $2 D G$-increased dedifferentiation, inflammatory reactions, and ER stress via the p38 kinase pathway. To identify the signaling pathway that GT is involved with in the regulation of $2 \mathrm{DG}$-induced dedifferentiation, inflammatory reactions, and ER-stress in chondrocytes, $\mathrm{SB}$ and $\mathrm{PD}$, the respective blockers of the $\mathrm{p} 38$ kinase and ERK-1/-2 pathways, and Salubrinal, a blocker of the ER-stress eIF2 $\alpha$ pathway, were used to pre-treat the cells for $2 \mathrm{~h}$. Then, western blotting was performed to verify the changes in the expression of type II collagen (a differentiated phenotype of chondrocytes), COX-2 (a major protein in inflammatory reactions), and GRP78 (an indicator of ER stress). Treatment of cells with Salubrinal (for blocking ER-stress eIF2 $\alpha$ signaling) resulted in reduced ER stress, while there were no changes in dedifferentiation or inflammatory reactions (Figs. 2A and 3A). Furthermore, treatment of cells with PD to block ERK-1/-2 signaling in fact retriggered COX-2 unglycosylation and increased ER stress. In addition, no changes related to dedifferentiation occurred (Figs. 2C and 3A). In contrast, treatment of cells with SB to block p38 kinase signaling caused changes in dedifferentiation, inflammatory reactions, and ER stress (Figs. 2B and 3A). While these changes were induced during transcription, COX-2 unglycosylation due to ER stress was considered to have occurred during protein translation or post-translational regulation and not during transcription (Fig. 3B). The statistical significance of the western blotting and RT-PCR expression data of type II collagen and COX-2 were quantified using ImageJ (Fig. 3C and D). To verify the results of the western blot analysis, alcian blue staining was used to quantify sulfated proteoglycan, another indicator of chondrocyte differentiation; the results were in conformity with that of the western blot analysis (Fig. 2D). Finally, immunofluorescence staining was used to monitor the cytological changes, and the findings were in agreement with the other results (Fig. 4). These results provided evidence that GT regulates 2DG-induced dedifferentiation, inflammatory responses, and ER stress via the p38 kinase pathway.

GT regulates $2 D G$-triggered dedifferentiation, inflammatory reactions, and ER stress via the ER-stress-induced p38 kinase pathway downstream from the IRE1 pathway. The findings of previous experiments revealed that GT regulates $2 \mathrm{DG}$-induced dedifferentiation, inflammatory responses, and ER stress through the p38 kinase pathway. This pathway was revealed to be a part of the IRE1 downstream signaling pathway among 
A

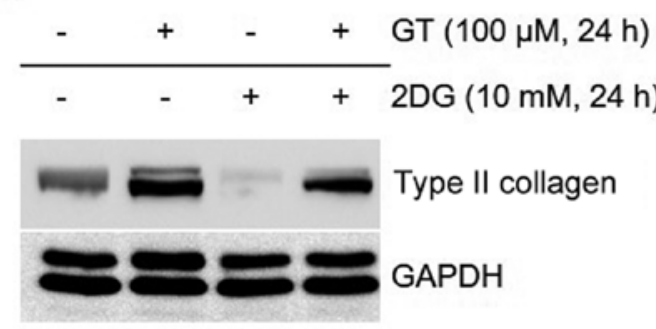

B

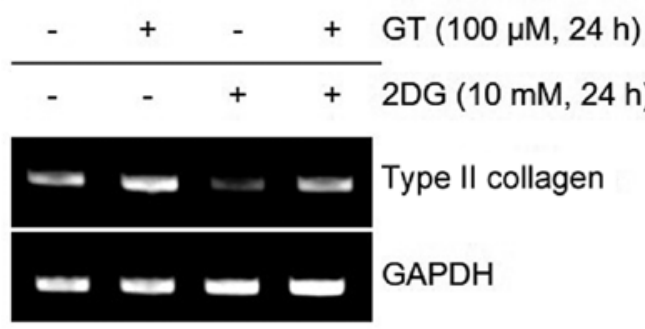

C \begin{tabular}{llll}
$-\quad+\quad+\quad$ & $+\mathrm{GT}(100 \mu \mathrm{M}, 24 \mathrm{~h})$ \\
\hline$-\quad+\quad+\quad$ & $+2 \mathrm{DG}(10 \mathrm{mM}, 24 \mathrm{~h})$
\end{tabular}

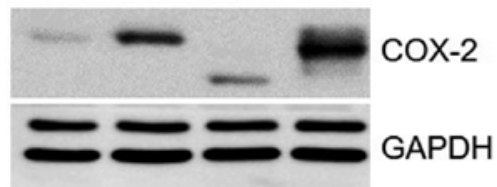

D

\begin{tabular}{|c|c|c|c|c|}
\hline - & + & - & + & GT $(100 \mu \mathrm{M}, 24 \mathrm{~h})$ \\
\hline - & - & + & + & 2DG (10 mM, 24 \\
\hline
\end{tabular}
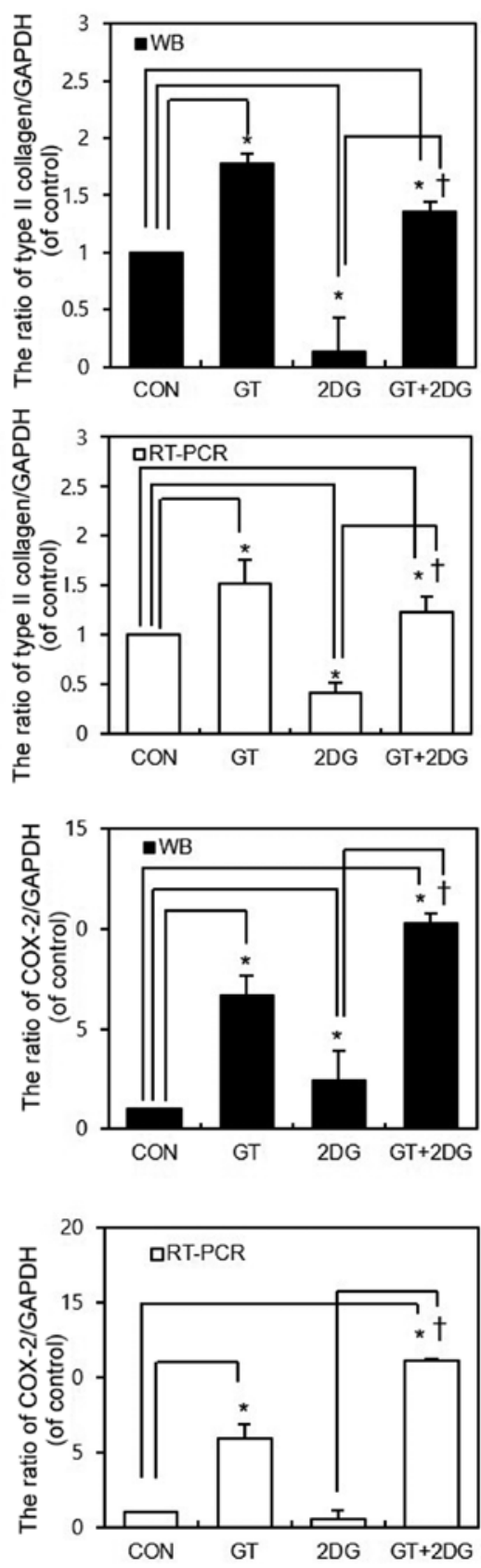

Figure 1. Effects of treatment of chondrocytes with GT and 2DG. (A-D) Chondrocytes were treated with $100 \mu \mathrm{M} \mathrm{GT}$ and $10 \mathrm{mM} 2 \mathrm{DG}$ for $24 \mathrm{~h}$. (A and B) Western blot and RT-PCR analyses were used to identify the expression of type II collagen and GAPDH. (C and D) Western blot and RT-PCR analyses were used to confirm expression of COX-2 and GAPDH. GAPDH was used as the loading control. (A-D) The ratio of type II collagen and COX-2/GAPDH. Results are presented as the means $\pm \mathrm{SD}$. ${ }^{*} \mathrm{P}<0.05$ compared with untreated cells, ${ }^{\dagger} \mathrm{P}<0.05$ compared with 2DG-treated cells. GT, gallotannin; 2DG, 2-deoxy-D-glucose; COX-2, cyclooxygenase- 2 .

the three signaling pathways triggered by ER stress. When the activities of all three signaling pathways were subsequently assessed, ATF6 and p-eIF2 $\alpha$ did not display any change, whereas IRE1, compared to the control, exhibited increased activity after a single treatment with a single 2DG dose and decreased activity with a single GT dose. We attempted to obtain eLF- $2 \alpha$ bands as a loading control to compare the expression of phosphorylated eLF- $2 \alpha$ bands due to reagent treatment. However, eLF-2 $\alpha$ bands could not be obtained due to a lack of immunoreactivity with a number of tested antibodies. In addition, after concurrent treatment with 2DG and GT, the IRE1 activity that was increased by 2DG was reduced by GT (Fig. 5A). This supported that GT is involved in the IRE1 signaling pathway in the inhibition of 2DG-induced ER stress. Furthermore, the changes in protein expression when IRE1 expression was reduced through IRE1 siRNA transfection were in concordance with the results of cells treated with SB to block the p38 kinase pathway (Fig. 5B). Lastly, it was examined whether splicing of XBP-1 mRNA was induced by the activated IRE1 downstream signaling pathway that was related to ER stress. It was revealed that, when compared to the control whereby no reagent was added, 2DG treatment induced splicing and increased XBP-1s, while GT treatment decreased XBP-1u. The concurrent treatment with GT and 
A

B

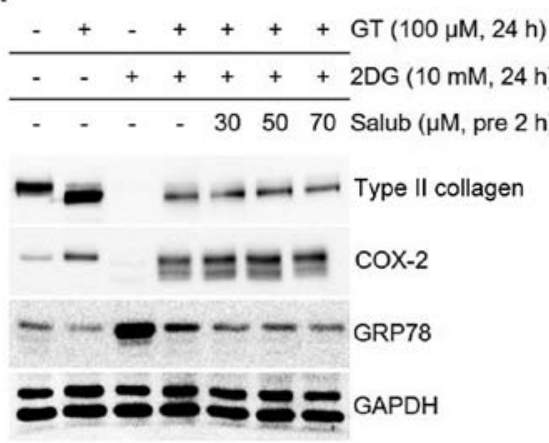

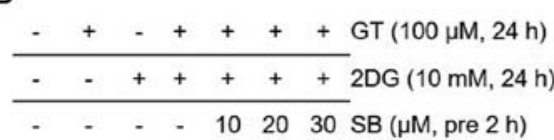

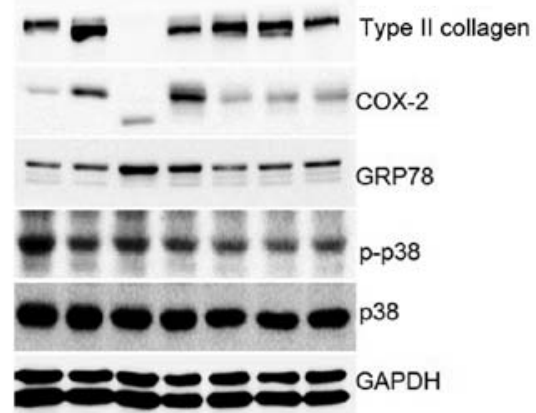

C
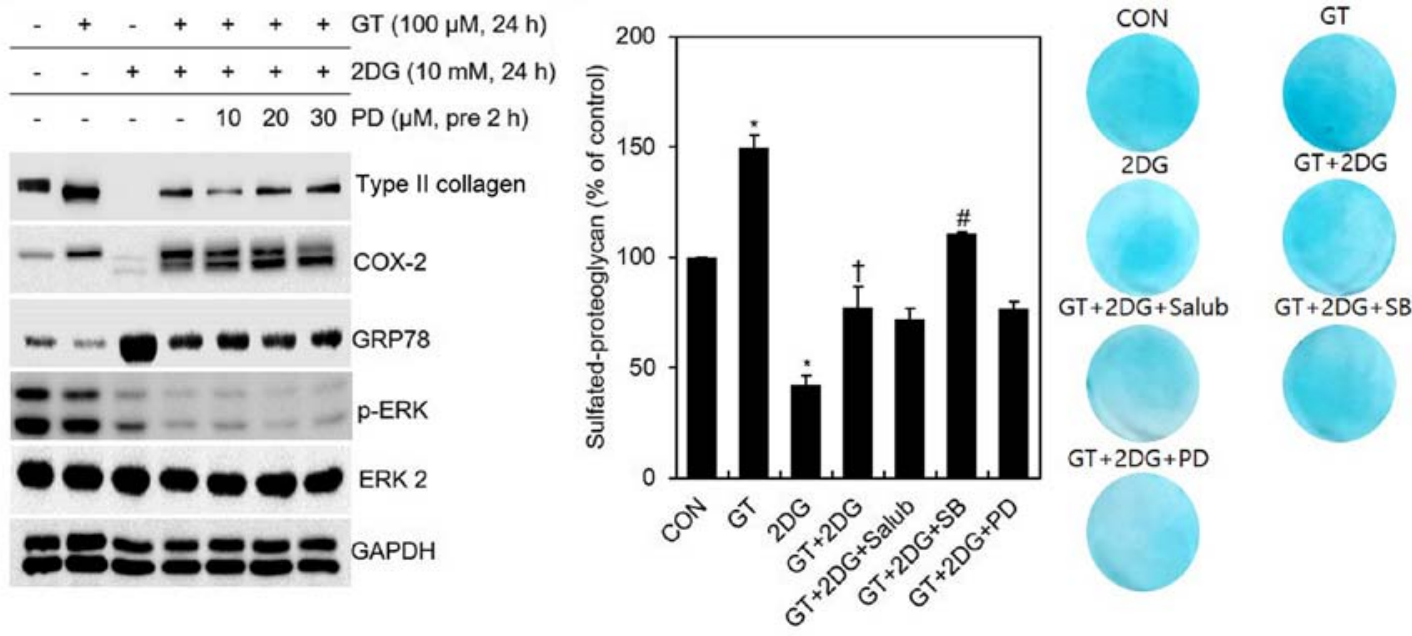

Figure 2. Identification of signaling pathways according to the blocker concentration after treatment of chondrocytes with GT and 2DG. (A-D) After pre-treatment for $2 \mathrm{~h}$ with specific concentrations of Salub, SB, or PD, chondrocytes were treated with $100 \mu \mathrm{M}$ GT and $10 \mathrm{mM} 2 \mathrm{DG}$ for $24 \mathrm{~h}$ After pre-treatment with a specific concentration of (A) Salub, (B) SB, or (C) PD, western blotting was used to determine the expression of type II collagen, COX-2, GRP78, p-p38, p-ERK and GAPDH. (D) Sulfated proteoglycan was examined using alcian blue staining. GAPDH, p38 and ERK 2 were used as the loading controls. Results are presented as the means $\pm \mathrm{SD}$. ${ }^{*} \mathrm{P}<0.05$ compared with untreated cells, ${ }^{\dagger} \mathrm{P}<0.05$ compared with $2 \mathrm{DG}$-treated cells, ${ }^{*} \mathrm{P}<0.05$ compared with GT $+2 \mathrm{DG}$-treated cells. GT, gallotannin; 2DG, 2-deoxy-D-glucose; Salub, salubrinal; COX-2, cyclooxygenase-2; GRP78, glucose-regulated protein 78 kDa.

2DG revealed that GT reduced the increased XBP-1s by 2DG-induced ER stress. The reduction of IRE1 expression after GT treatment or IRE1 siRNA transfection was accompanied by a decreased expression of XBP-1 mRNA (Fig. 5C). These findings provided evidence that GT regulates $2 \mathrm{DG}$-induced dedifferentiation, inflammatory reactions, and ER stress via the ER-stress-induced p38 kinase pathway downstream from the IRE1 pathway.

\section{Discussion}

The number of patients with $\mathrm{OA}$ is continuously increasing due to aging and prolonged human lifespan. However, inadequate research and current therapeutic development warrant the need for more studies. Cartilage degeneration leads to OA. Among the various causes of OA, the most recognized is metabolic imbalance (2). Metabolic imbalance, as a representative cause of OA, may result from the intracellular accumulation of 2DG, a glucose derivative with $2^{\prime} \mathrm{OH}$ replaced by an $\mathrm{H}$, which inhibits normal glycolysis and glycosylation to perturb the metabolic balance and induce ER stress, thereby inducing dedifferentiation in chondrocytes $(20,22)$. In contrast, GT, a hydrolyzable tannin and a plant polyphenol, is used as a protective agent in a similar manner to a drug, and exhibits high tolerance against cancers, oxidation, viruses, bacteria, and parasites by promoting differentiation of chondrocytes (21). Although the independent effects of 2DG and GT in chondrocytes have been examined in previous studies, the effects of concurrent treatment with GT and 2DG, as well as signaling pathways regulating their activities, have not been adequately investigated. Thus, the present study was performed to verify whether GT works against 2DG to promote differentiation of chondrocytes. This study also identified the signaling pathways involved in this regulation.

When chondrocytes were treated with both GT and 2DG, type II collagen expression, which was otherwise reduced by $2 \mathrm{DG}$, was enhanced. The type II collagen band shifts are slightly different depending on the experimental conditions. Type II collagen is predominant and consists of approximately $80 \%$ of total vitreous collagen. Possibly it depends on the 
A

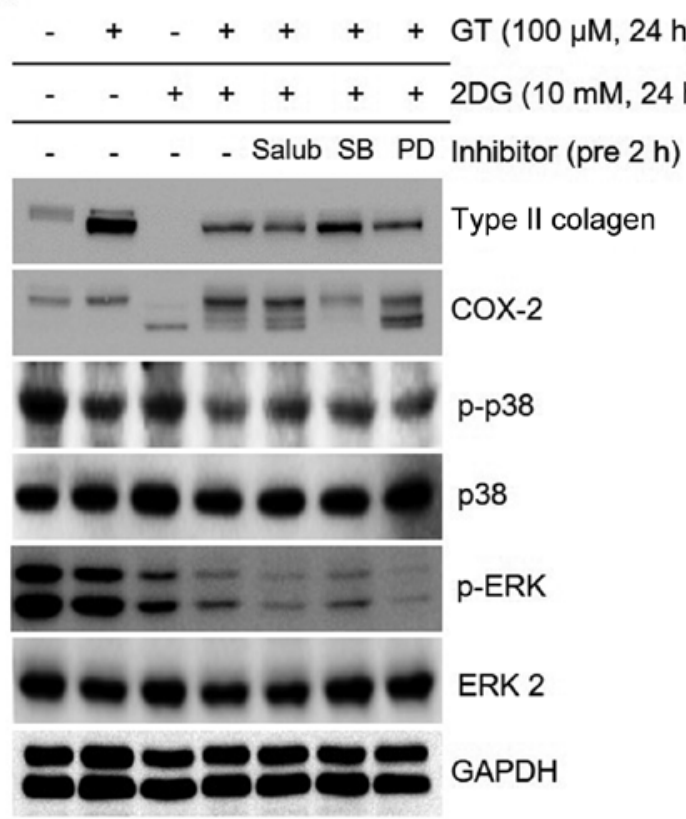

B

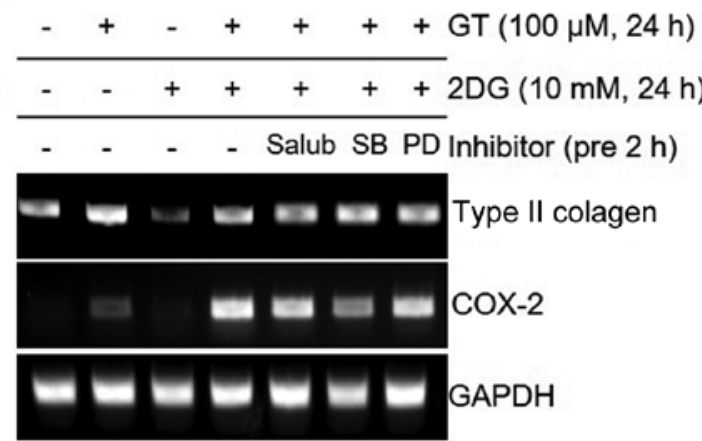
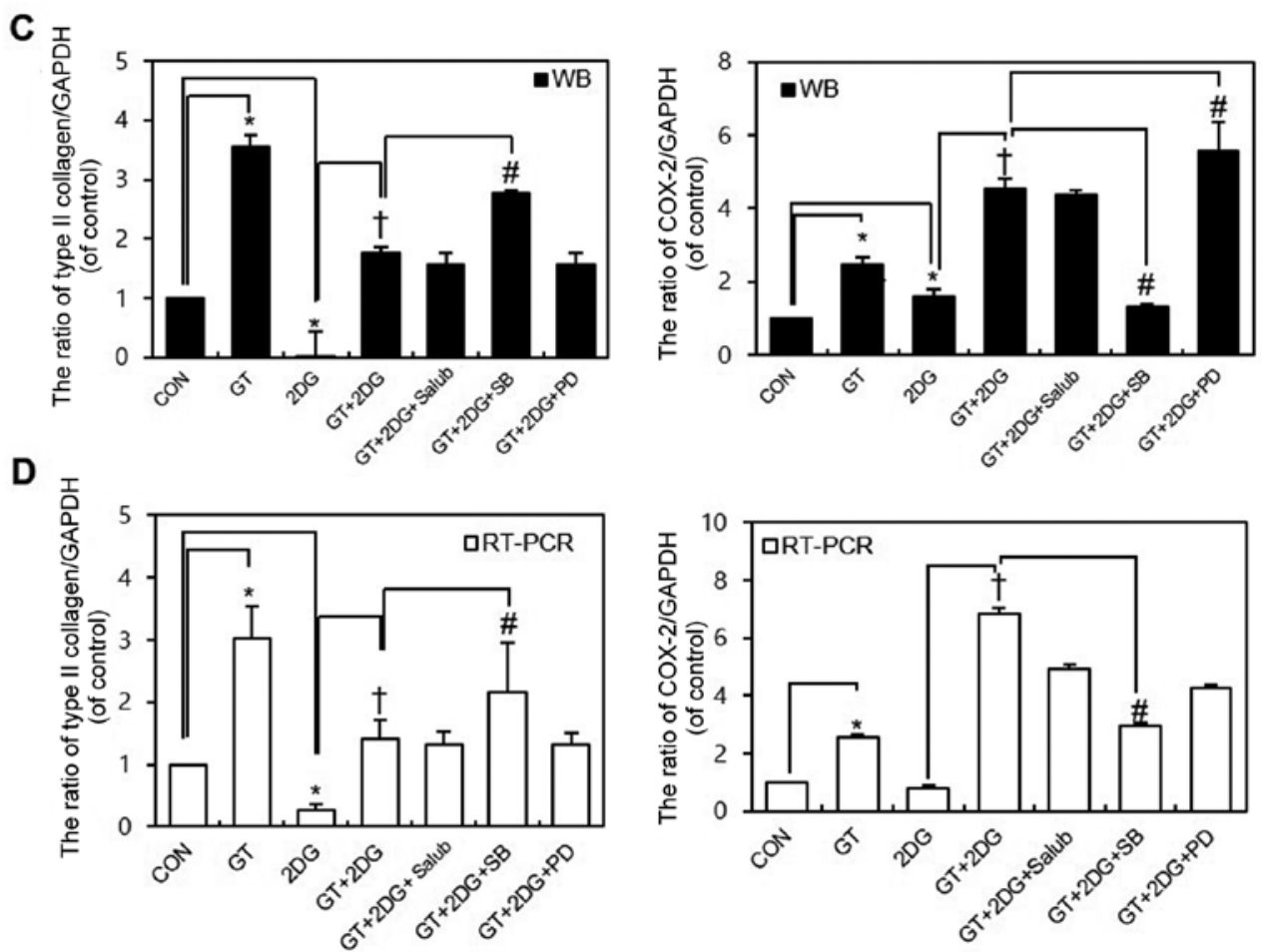

Figure 3. Identification of signaling pathways according to the blocker after treatment of chondrocytes with GT and 2DG. (A and B) After pre-treating chondrocytes for $2 \mathrm{~h}$ with specific concentrations of Salub, SB, or PD, chondrocytes were treated with $100 \mu \mathrm{M}$ GT and $10 \mathrm{mM} 2 \mathrm{DG}$ for $24 \mathrm{~h}$. (A) Western blotting was used to determine the expression of type II collagen, COX-2, p-p38, p-ERK, and GAPDH. (B) RT-PCR was used to determine the expression of type II collagen, COX-2, and GAPDH. p38, ERK 2 and GAPDH was used as the loading control. (C and D) Relative expression (\% of control) of type II collagen and COX-2. Results are presented as the means $\pm \mathrm{SD}$. ${ }^{*} \mathrm{P}<0.05$ compared with untreated cells, ${ }^{\circ} \mathrm{P}<0.05$ compared with $2 \mathrm{DG}$-treated cells, ${ }^{\prime \prime} \mathrm{P}<0.05$ compared with GT + 2DG-treated cells. GT, gallotannin; 2DG, 2-deoxy-D-glucose; Salub, salubrinal; COX-2, cyclooxygenase-2; GRP78, glucose-regulated protein 78 kDa .

enzymatic degradation of extracellular matrix. Similarly, GT promoted COX-2 expression that was otherwise reduced by 2DG, and prevented unglycosylation caused by 2DG-induced ER stress (Fig. 1). These findings provide evidence that GT attenuates 2DG-induced dedifferentiation and ER stress and regulates inflammatory reactions. To demonstrate the role of GT in attenuating ER stress, the reduced expression of GRP78 as an indicator of ER stress (15) was observed
(Figs. 2 and 4). To identify the signaling pathway involved in GT attenuating 2DG-induced dedifferentiation and ER stress and regulation of inflammatory reactions, chondrocytes were treated with SB and PD, blockers of the p38 kinase and ERK-1/-2 pathways, respectively, during differentiation and inflammation, and with Salubrinal, a blocker of the ER-stress eIF2 $\alpha$ pathway (25). Among these pathways, inhibition of the p38 kinase pathway resulted in an increased expression of 

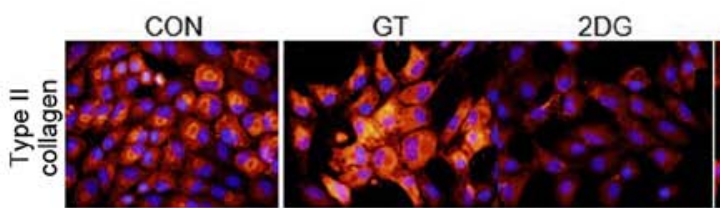
$\mathrm{GT}+2 \mathrm{DG}$ $\mathrm{GT}+2 \mathrm{DG}+$ Salub
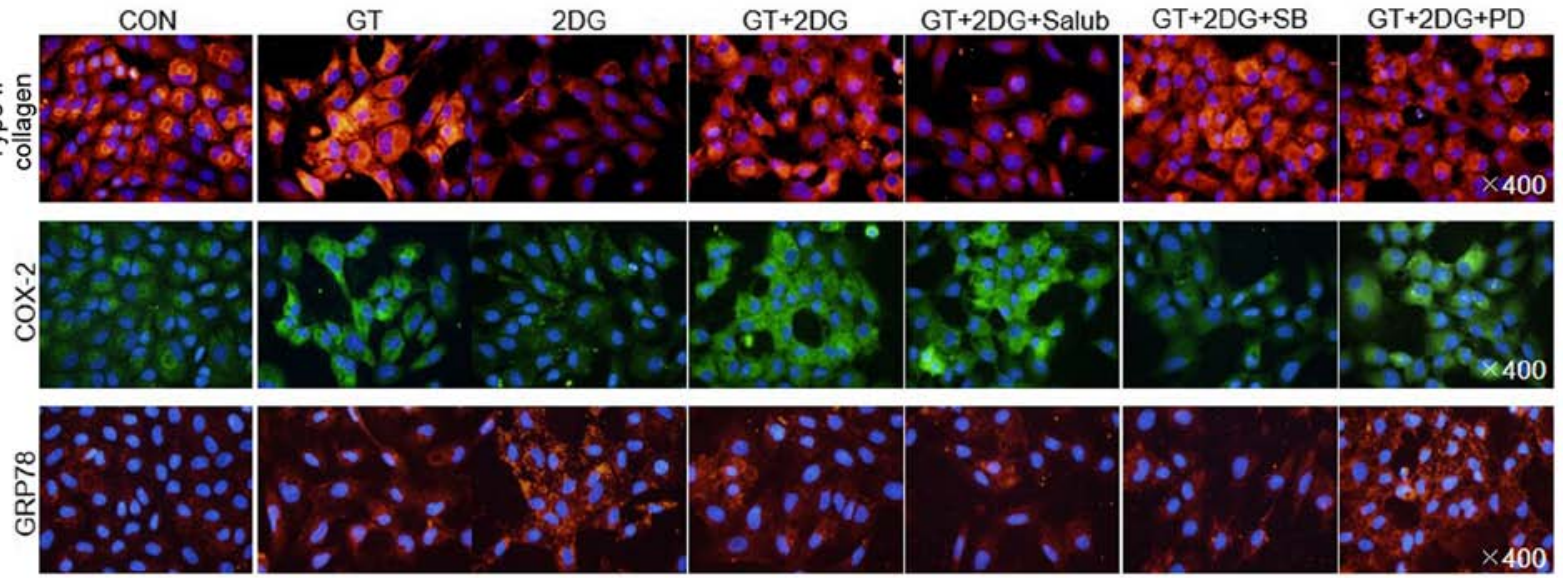

Figure 4. Identification of signaling pathways using immunofluorescence after treatment of chondrocytes with GT and 2DG. After pre-treating chondrocytes for $2 \mathrm{~h}$ with specific concentrations of Salub, SB, or PD, chondrocytes were treated with $100 \mu \mathrm{M}$ GT and $10 \mathrm{mM} 2 \mathrm{DG}$ for $24 \mathrm{~h}$. Immunofluorescence microscopy (magnification, $\mathrm{x} 400$ ) was used to determine the expression of type II collagen, COX-2, and GRP78. The cellular nuclei were stained with DAPI. GT, gallotannin; 2DG, 2-deoxy-D-glucose; Salub, salubrinal; COX-2, cyclooxygenase-2; GRP78, glucose-regulated protein $78 \mathrm{kDa}$.

A

\begin{tabular}{ccc}
$-\quad+\quad+\quad+\quad+\quad$ & GT $(100 \mu \mathrm{M}, 24 \mathrm{~h})$ \\
\hline$-\quad 2 D G(10 \mathrm{mM}, 24 \mathrm{~h})$
\end{tabular}

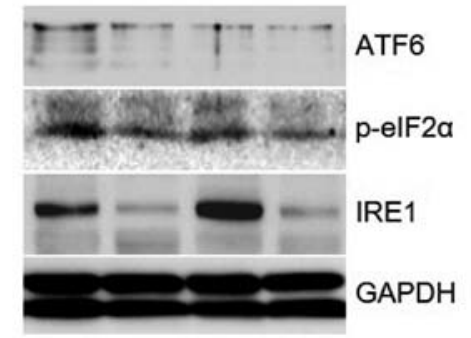

C

\begin{tabular}{|c|c|c|c|c|c|}
\hline - & + & - & + & + & GT $(100 \mu \mathrm{M}, 24 \mathrm{~h})$ \\
\hline- & - & + & + & + & 2DG (10 mM, $24 \mathrm{~h})$ \\
\hline - & - & - & - & + & $\begin{array}{l}\text { IRE1 siRNA } \\
\text { (150 nM, pre } 10 \mathrm{~h})\end{array}$ \\
\hline+ & + & + & + & - & $\begin{array}{l}\text { Scrambled siRNA } \\
\text { (150 nM, pre } 10 \mathrm{~h})\end{array}$ \\
\hline ש & E & $=$ & 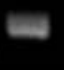 & a & $\begin{array}{l}\leftarrow \text { XBP-1u } \\
\leftarrow \text { XBP-1s }\end{array}$ \\
\hline & & & & & GAPDH \\
\hline
\end{tabular}

B

\begin{tabular}{|c|c|c|c|c|c|c|}
\hline - & + & - & + & & & GT $(100 \mu \mathrm{M}, 24 \mathrm{~h})$ \\
\hline- & - & + & + & + & - & 2DG (10 mM, $24 \mathrm{~h})$ \\
\hline - & - & - & - & + & + & $\begin{array}{l}\text { IRE1 siRNA } \\
(150 \mathrm{nM} \text {, pre } 10 \mathrm{~h})\end{array}$ \\
\hline+ & + & & + & - & 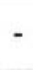 & $\begin{array}{l}\text { Scrambled siRNA } \\
(150 \mathrm{nM}, \text { pre } 10 \mathrm{~h})\end{array}$ \\
\hline
\end{tabular}

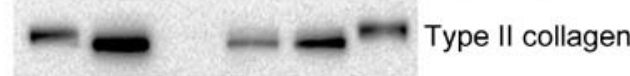

$-\pi-\operatorname{cox}-2$
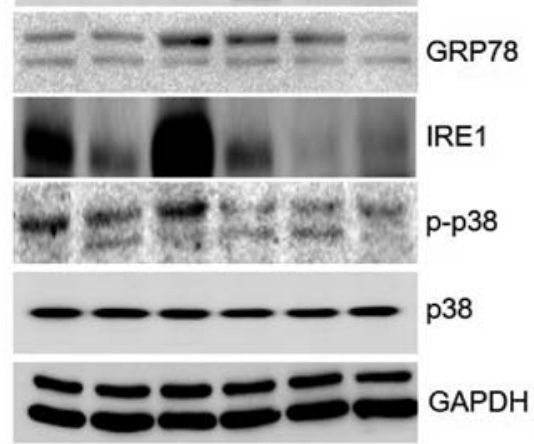

Figure 5. Identification of signaling pathways after treatment of chondrocytes with GT and 2DG. (A and B) Chondrocytes were treated with $100 \mu \mathrm{M}$ GT and $10 \mathrm{mM} 2 \mathrm{DG}$ for $24 \mathrm{~h}$. (A) Western blotting was used to determine the expression of ATF6, p-eIF2 $\alpha$, and IRE1. (B) Western blot was used to confirm expression of type II collagen, COX-2, GRP78, IRE1, p-p38, and GAPDH for the chondrocytes that had undergone transfection with $150 \mathrm{nM}$ IRE1 siRNA. (C) RT-PCR was used to verify the expression of XBP-1 and GAPDH. p38 and GAPDH were used as the loading controls and scrambled siRNA was used as the negative control. GT, gallotannin; 2DG, 2-deoxy-D-glucose; ATF6, activating transcription factor 6; IRE1, inositol-requiring enzyme 1; COX-2, cyclooxygenase-2; GRP78, glucose-regulated protein $78 \mathrm{kDa}$; XBP-1, X-box binding protein 1.

type II collagen and COX-2 expression was further decreased, indicating that the p38 kinase pathway was influenced by GT (Figs. 2-4). These findings thus provide evidence that GT attenuates 2DG-induced dedifferentiation, inflammatory reactions, and ER stress through the p38 kinase pathway. Furthermore, after having confirmed that IRE1 downstream signaling pathways involve the p38 kinase pathway among the three signaling pathways activated by ER stress, the activities of all three signaling pathways were assessed. ATF6 and p-eIF2 $\alpha$ did not display changes in their activities, whereas IRE1 activity that was otherwise increased by 2DG was suppressed by GT. The subsequent experiment using IRE1 siRNA transfection produced results identical to those when the p38 kinase pathway was inhibited; type II collagen expression was further enhanced and COX-2 expression was further reduced. Moreover, the splicing of XBP-1 mRNA, 
which was caused by the ER-stress-related IRE1 downstream signaling pathway, confirmed that GT reduced the increased XBP-1s due to 2DG-induced ER stress. In addition, IRE1 expression was reduced after GT treatment and IRE1 siRNA transfection accompanied the reduced XBP-1 mRNA expression (Fig. 5), suggesting that IRE1 had an influence on the expression of XBP-1 mRNA; however, further studies are required to confirm these findings. The present results thus provide evidence that GT regulates 2DG-induced dedifferentiation, inflammatory reactions, and ER stress via the ER-stress-induced p38 kinase pathway downstream from the IRE1 pathway.

The findings aggregately support that GT inhibits 2DG-induced dedifferentiation and ER-stress-induced COX-2 unglycosylation in chondrocytes. GT was also revealed to regulate differentiation and inflammation via the ER-stress-induced p38 kinase pathway downstream from the IRE1 pathway. These effects of GT on dedifferentiation and ER stress suggest that GT could be utilized in the therapeutic treatment of arthritis. Furthermore, based on our in vitro experimental results, further in vivo experiments should be performed to provide fundamental data and generate concrete evidence for treating arthritis using chondrocytes.

\section{Acknowledgements}

Not applicable.

\section{Funding}

The present study was supported by grants from the Kongju National University.

\section{Availability of data and materials}

The datasets used and/or analyzed during the current study are available from the corresponding author on reasonable request.

\section{Authors' contributions}

SMK, YH, SMY and SJK designed the experiments, conducted the study, analyzed the data and wrote the manuscript. All authors read and approved the manuscript and agree to be accountable for all aspects of the research in ensuring that the accuracy or integrity of any part of the work are appropriately investigated and resolved.

\section{Ethics approval and consent to participate}

The research conducted in the present study was approved by the Ethics Committee of Kongju National University.

\section{Patient consent for publication}

Not applicable.

\section{Competing interests}

The authors declare that they have no competing interests.

\section{References}

1. Bian Q, Wang YJ, Liu SF and Li YP: Osteoarthritis: Genetic factors, animal models, mechanisms, and therapies. Front Biosci (Elite Ed) 4: 74-100, 2012.

2. Kim JH, Jeon J, Shin M, Won Y, Lee M, Kwak JS, Lee G, Rhee J, Ryu JH, Chun $\mathrm{CH}$ and Chun JS: Regulation of the catabolic cascade in osteoarthritis by the zinc-ZIP8-MTF1 axis. Cell 156: 730-743, 2014.

3. Chen JL, Duan L, Zhu W, Xiong J and Wang D: Extracellular matrix production in vitro in cartilage tissue engineering. J Transl Med 12: 88, 2014.

4. Eyre D: Collagen of articular cartilage. Arthritis Res 4: 30-35, 2002.

5. Chandrasekharan NV and Simmons DL: The cyclooxygenases. Genome Biol 5: 241, 2004.

6. Pang LY, Hurst EA and Argyle DJ: Cyclooxygenase-2: A role in cancer stem cell survival and repopulation of cancer cells during therapy. Stem Cells Int 2016: 2048731, 2016.

7. Herschman HR, Reddy ST and Xie W: Function and regulation of prostaglandin synthase-2. Adv Exp Med Biol 407: 61-66, 1997.

8. Gronowski AM and Rotwein P: Rapid changes in nuclear protein tyrosine phosphorylation after growth hormone treatment in vivo. Identification of phosphorylated mitogen-activated protein kinase and STAT91. J Biol Chem 269: 7874-7878, 1994.

9. Pelech SL and Sanghera JS: Mitogen-activated protein kinases: Versatile transducers for cell signaling. Trends Biochem Sci 17: 233-238, 1992.

10. Cargnello $M$ and Roux PP: Activation and function of the MAPKs and their substrates, the MAPK-activated protein kinases. Microbiol Mol Biol Rev 75: 50-83, 2011.

11. Roux PP and Blenis J: ERK and p38 MAPK-activated protein kinases: A family of protein kinases with diverse biological functions. Microbiol Mol Biol Rev 68: 320-344, 2004.

12. Schwarz DS and Blower MD: The endoplasmic reticulum: Structure, function and response to cellular signaling. Cell Mol Life Sci 73: 79-94, 2016.

13. Ellgaard L and Helenius A: Quality control in the endoplasmic reticulum. Nat Rev Mol Cell Biol 4: 181-191, 2003.

14. Zhang K and Kaufman RJ: Protein folding in the endoplasmic reticulum and the unfolded protein response. Handb Exp Pharmacol 2006: 69-91, 2006.

15. Ni M, Zhang Y and Lee AS: Beyond the endoplasmic reticulum: Atypical GRP78 in cell viability, signalling and therapeutic targeting. Biochem J 434: 181-188, 2011.

16. Ron D and Walter P: Signal integration in the endoplasmic reticulum unfolded protein response. Nat Rev Mol Cell Biol 8: 519-529, 2007.

17. Hetz C, Martinon F, Rodriguez D and Glimcher LH: The unfolded protein response: Integrating stress signals through the stress sensor IRE1 $\alpha$. Physiol Rev 91: 1219-1243, 2011.

18. Lee AH, Iwakoshi NN and Glimcher LH: XBP-1 regulates a subset of endoplasmic reticulum resident chaperone genes in the unfolded protein response. Mol Cell Biol 23: 7448-7459, 2003.

19. Zhang K and Kaufman RJ: The unfolded protein response: A stress signaling pathway critical for health and disease. Neurology 66 (2 Suppl 1): S102-S109, 2006.

20. Yu SM and Kim SJ: Endoplasmic reticulum stress (ER-stress) by 2-deoxy-D-glucose (2DG) reduces cyclooxygenase-2 (COX-2) expression and $\mathrm{N}$-glycosylation and induces a loss of COX-2 activity via a Src kinase-dependent pathway in rabbit articular chondrocytes. Exp Mol Med 42: 777-786, 2010.

21. Lee WK, Chung KW, Kim GH and Kim SJ: Gallotannin causes differentiation and inflammation via ERK-1/-2 and p38 kinase pathways in rabbit articular chondrocytes. Mol Med Rep 7: 701-707, 2013.

22. Yu SM, Kim HA and Kim SJ: 2-Deoxy-D-glucose regulates dedifferentiation through beta-catenin pathway in rabbit articular chondrocytes. Exp Mol Med 42: 503-513, 2010.

23. Korean academy of nursing: 'Ether anesthesia'. The great encyclopedia of nursing science. Korea dictionary research publishing: 1-1951, 1996.

24. Eo SH, Kim DW, Choi SY, Kim HA and Kim SJ: PEP-1-SIRT2 causes dedifferentiation and COX-2 expression via the MAPK pathways in rabbit articular chondrocytes. Exp Cell Res 339: 351-359, 2015.

25. Darling NJ and Cook SJ: The role of MAPK signalling pathways in the response to endoplasmic reticulum stress. Biochim Biophys Acta 1843: 2150-2163, 2014. 